\title{
Harmonized Cross-Sectional Surveys Focused on Fluid Intake in Children, Adolescents and Adults: The Liq.In ${ }^{7}$ Initiative
}

\author{
Homero Martinez ${ }^{a, b} \quad$ Isabelle Guelinckx ${ }^{c} \quad$ Jordi Salas-Salvadód, e \\ Joan Gandyg, h Stavros A. Kavouras ${ }^{i}$ Luis A. Moreno ${ }^{e, f}$ \\ ${ }^{a}$ Micronutrient Forum, Micronutrient Initiative, Ottawa, Ont., Canada; ${ }^{b}$ Hospital Infantil de Mexico Federico \\ Gomez, Mexico City, Mexico; ${ }^{\mathrm{C} H y d r a t i o n}$ and Health Department, Danone Research, Palaiseau, France; ${ }^{\mathrm{d}}$ Human \\ Nutrition Unit, Hospital Universitari de Sant Joan de Reus, Faculty of Medicine and Health Sciences, IISPV (Institut \\ d'Investigació Sanitària Pere Virgili), Biochemistry Biotechnology Department, Universitat Rovira i Virgili, Reus, \\ e CIBERobn (Centro de Investigación Biomédica en Red Fisiopatología de la Obesidad y Nutrición), Institute of \\ Health Carlos III, Madrid, and fGENUD (Growth, Exercise, NUtrition and Development) Research Group, Faculty of \\ Health Sciences, Universidad de Zaragoza, Zaragoza, Spain; ${ }^{9}$ British Dietetic Association, Birmingham, and ${ }^{\mathrm{h}}$ School \\ of Life and Medical Services, University of Hertfordshire, Hatfield, UK; i Department of Health, Human Performance \\ and Recreation, University of Arkansas, Fayetteville, Ark., USA
}

\section{Key Words}

Water · Beverages · Fluid intake $\cdot$ Liq. $\operatorname{In}^{7} \cdot 7$-day fluid diary · Hydration

\begin{abstract}
Objective: To assess the intake of water and all other beverages in children, adolescents and adults. Methods: Three thousand six hundred eleven children ( $8 \pm 2$ years), 8,109 adolescents ( $13 \pm 2$ years) and 16,276 adults ( $40 \pm 14$ years) ( $47 \%$ men) were recruited in 15 cross-sectional surveys (liquid intake across 7 days, Liq. In $^{7}$ study) and completed a 7-day fluid-specific record to assess total fluid intake (TFI), where TFI was defined as the sum of drinking water and other type of beverages. Results: The median TFI was 1.2, 1.2 and 1.8 liters/day in children, adolescents and adults respectively, with important differences observed between countries. Only $39 \%$ of children, $25 \%$ of adolescents and $51 \%$ of adults met the European Food Safety Authority adequate intake (AI) recommendations of water from fluids. In the surveys of Spain, France, Belgium, Germany, Turkey, Iran, Indonesia and China, water was the major contributor (47-78\%) to TFI.
\end{abstract}

\section{KARGER}

E-Mail karger@karger.com www.karger.com/anm

\section{(c) 2016 The Author(s) \\ Published by S. Karger AG, Basel 0250-6807/16/0686-0012\$39.50/0}

This article is licensed under the Creative Commons AttributionNonCommercial-NoDerivatives 4.0 International License (CC BYNC-ND) (http://www.karger.com/Services/OpenAccessLicense) Usage and distribution for commercial purposes as well as any distribution of modified material requires written permission.
In the adult surveys of UK, Poland, Japan and Argentina, hot beverages were the highest contributor to TFI. The fluid intake of children and adolescents in Mexico, Brazil, Argentina and Uruguay was characterized by a contribution of juices and sweet beverages that was as important as the contribution of water to TFI. Conclusion: Given that a relatively high proportion of subjects, especially children and adolescents, failed to meet the recommended Al of water from fluids and that water intake was not the highest contributor to TFI in all countries, undertaking actions to increase water intake are warranted.

(c) 2016 The Author(s)

Published by S. Karger AG, Basel

\section{Introduction}

In the last decade, epidemiological evidence has linked total fluid intake (TFI, the sum of drinking water and all other beverages) and the intake of water and/or other beverages to different health outcomes. Preliminary evidence indicated that increased consumption of water has the potential to reduce energy intake and the risk of obesity [1, 
2], urinary tract infections [3] and the incidence of hyperglycemia [4]. Prospective and cross-sectional analyses of different cohorts have shown that the likelihood of developing chronic kidney disease (CKD) was reduced in those participants with a high TFI [5-7]. An analysis of the 2005-2006 National Health and Nutrition Examination Survey data moreover confirmed an inverse association between CKD prevalence and the amount of plain water intake; however, this association did not exist with beverages other than plain water [7]. Indeed, a high water intake has been associated with CKD risk reduction, while several meta-analyses associated a high daily intake of sugared beverages with a CKD risk increase. Compared with infrequent sugared beverage consumers ( $<1$ serving/week or $\leq 1$ serving/month), individuals consuming on average $\geq 1$ servings of $250 \mathrm{ml} /$ day of sugared beverages had a higher risk of developing kidney stones, metabolic syndrome, type 2 diabetes, obesity and hypertension [8-11].

However, the relevance of this research for public health is not often shown due to the paucity of fluid intake data. Recently, publications have reviewed existing food surveys with a specific focus on drinking water intake and beverages of all types $[12,13]$, or have provided original fluid intake data [14]. These reviews however illustrated that study designs, dietary assessment methods or classifications of beverages and age categories varied; these differences limit the comparison of results between studies or countries. Moreover, only $50 \%$ of the 38 surveys included in the review by Özen et al. [12] reported the intake of drinking water. Therefore, the aim of the crosssectional surveys summarized here was to assess the intake of drinking water and other beverages with a harmonized protocol, in different samples of children, adolescents and adults.

\section{Methods}

The present article summarizes published data that was collected in 15 different cross-sectional surveys involving 3,611 children (4-9 years), 8,109 adolescents (10-17 years) and 16,276 adults ( $\geq 18$ years). The surveys were conducted between 2008 and 2014 in South America (Mexico, Brazil, Uruguay and Argentina), Europe (Spain, France, Belgium, United Kingdom, Germany, Poland and Turkey) and Asia (Iran, China, Indonesia and Japan). Participants were randomly recruited from a database of volunteers and/or via a systematic door-to-door recruitment, until the quotas for age, gender, region, habitat and/or socioeconomic characteristics (i.e., in relation to the total country population) were met. Detailed protocol descriptions and results of these 15 surveys have been published elsewhere [15-24]. The survey protocol of the unpublished surveys was reviewed and approved by the University of Arkansas Review Board (ref. 14-12-376).

Fluid Intake of Children, Adolescents and Adults
Participants were provided with a fluid-specific record to collect information related to their intakes of all fluid types during 7 consecutive days. The information included the type of beverage, the volume consumed and where and when the fluid was consumed. An identical 7-day fluid-specific record was completed either online or on paper. To increase the reported accuracy of the volume of fluid consumed by the participants, a photographic booklet of standard fluid containers was provided. All the 15 surveys used this method to assess the fluid intake and were referred to as liquid intake across 7 days (Liq. $\operatorname{In}^{7}$ ).

Fluids were classified as water (tap and bottled water), milk and milk derivatives, hot beverages (coffee, tea and other hot beverages), juices, sweet beverages (carbonated and non-carbonated soft drinks, energy drinks, sports drinks and other sugared soft drinks), diet beverages (diet carbonated soft drinks, diet non-carbonated soft drinks and other diet soft drinks), alcoholic drinks and other beverages. In the children and adolescent sample, as well as in the adult samples of UK, Poland, Indonesia and Japan, the intake of diet beverages was very small; therefore, during the first data treatment, they were included in the sweet beverage category. In Argentina, Iran and Indonesia, alcoholic beverages were not recorded, and in Belgium, hot beverages were not recorded. TFI was defined as the sum of all these categories. To evaluate the adequacy of fluid intake, the European Food Safety Authority (EFSA) age- and gender-specific adequate intakes (AIs) for total water intake were used, after subtracting $20 \%$ estimated to correspond to water intake from foods [25].

\section{Results}

Figure 1 describes the mean total water intake for all age groups by country. On an average, children (mean age $8 \pm 2$ years) consumed the recommended amount of TFI (1.3 liters/day), with the exception of France $(1,018$ $\mathrm{ml} /$ day), China ( $966 \mathrm{ml} /$ day) and Belgium ( $839 \mathrm{ml} /$ day). Combining both genders, fluid intake by adolescents (mean age $13 \pm 2$ years) failed to meet the recommended AI (1.7-2 liters/day for boys and 1.5-2 liters/day for girls) in Mexico (1,509 ml/day), France (1,253 ml/day), Belgium ( $948 \mathrm{ml} /$ day), Poland (1,476 ml/day), Iran (1,320 $\mathrm{ml} /$ day) and China $(1,153 \mathrm{ml} /$ day $)$. Adult participants (mean age $40 \pm 14$ years) met the recommended AI of water (2.0 liters/day for males and 1.6 liters/day for females) apart from in France $(1,545 \mathrm{ml} /$ day). For the entire sample (data not shown), the mean TFI was 1.2 liters/day in children, 1.2 liters/day in adolescents and 1.8 liters/day in adults. Only $39 \%$ of children, $25 \%$ of adolescents and $53 \%$ of adults met the EFSA AI of water from fluids.

Figure 2 illustrates the contributions of water and the different beverages to TFI, per country and age group. In 8 out of the 15 countries, water represented half or more (44-79\%) of TFI in all age groups. In the adult samples of Poland, UK, Argentina and Japan, hot beverages were the main contributors to TFI. In the children and adolescent 
Water $\square$ Milk and derivates $\square$ Hot beverages $\square$ Juices $\square$ Sweet beverages $\square$ Diet beverages $\square$ Alcoholic beverages $\square$ Other beverages

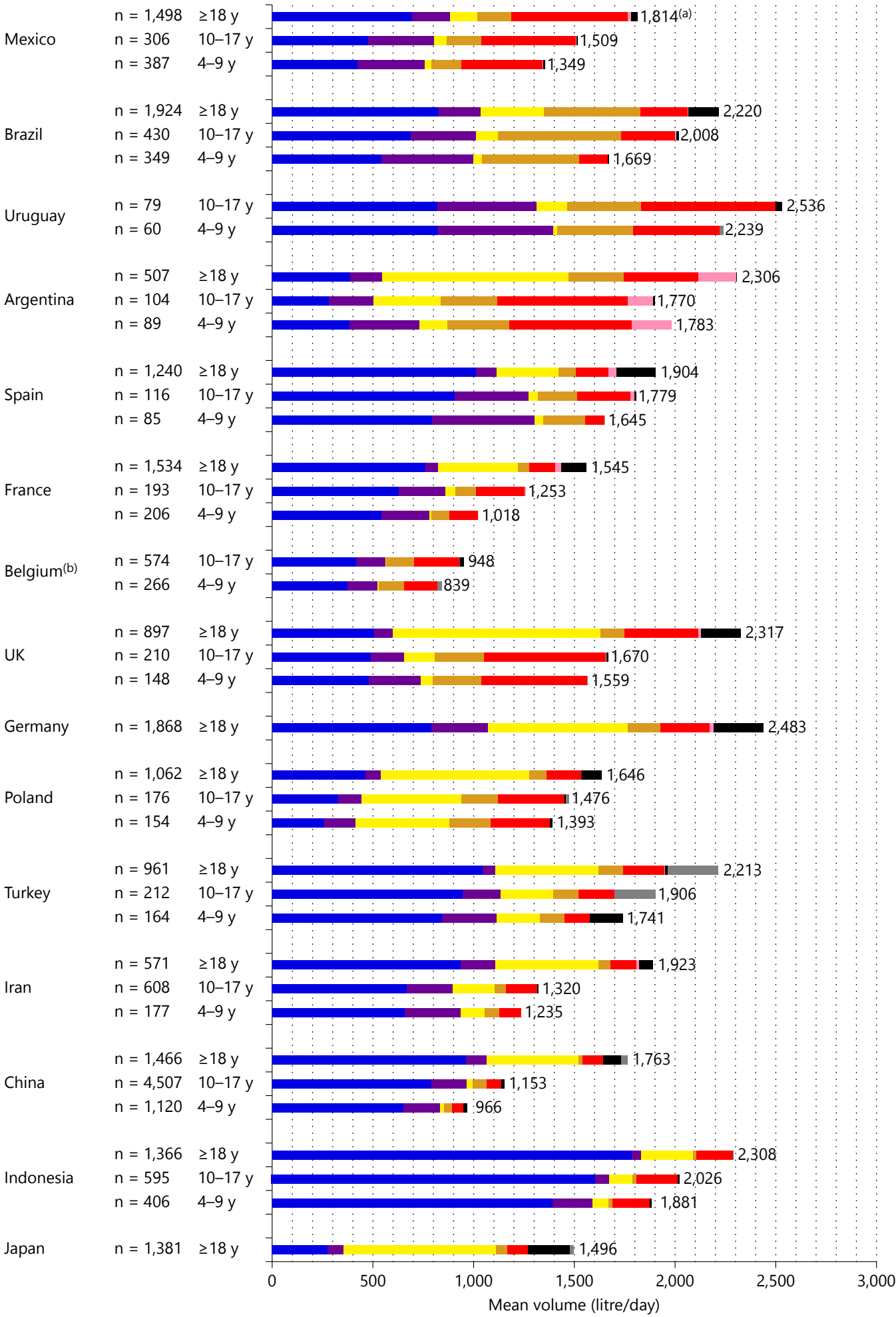

Fig. 1. Mean TFI (ml/day) and intakes (ml/day) of water and other beverages according to age group and country. ${ }^{\text {a }}$ The mean TFI of each demographic group is shown numerically; ${ }^{\mathrm{b}}$ hot beverages were not recorded in the Belgian sample. $y=$ Years. 


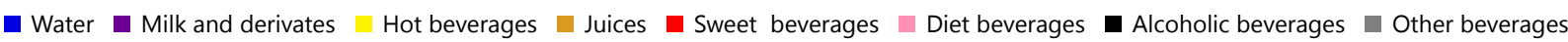

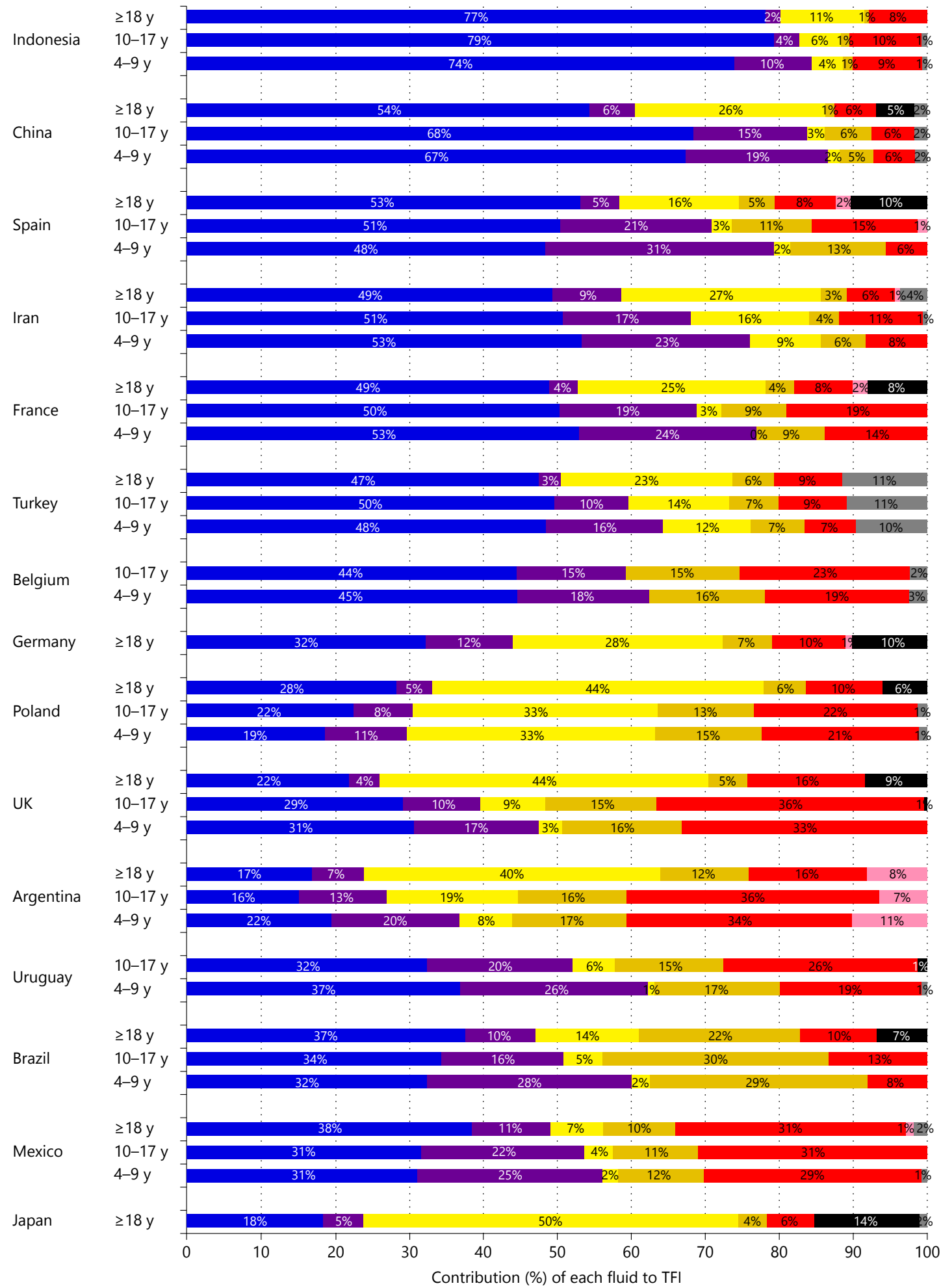

Fig. 2. Contribution (\%) of each fluid type to TFI according to age group and country. $y=$ Years.

Fluid Intake of Children, Adolescents and Adults
Ann Nutr Metab 2016;68(suppl 2):12-18

DOI: $10.1159 / 000446199$ 


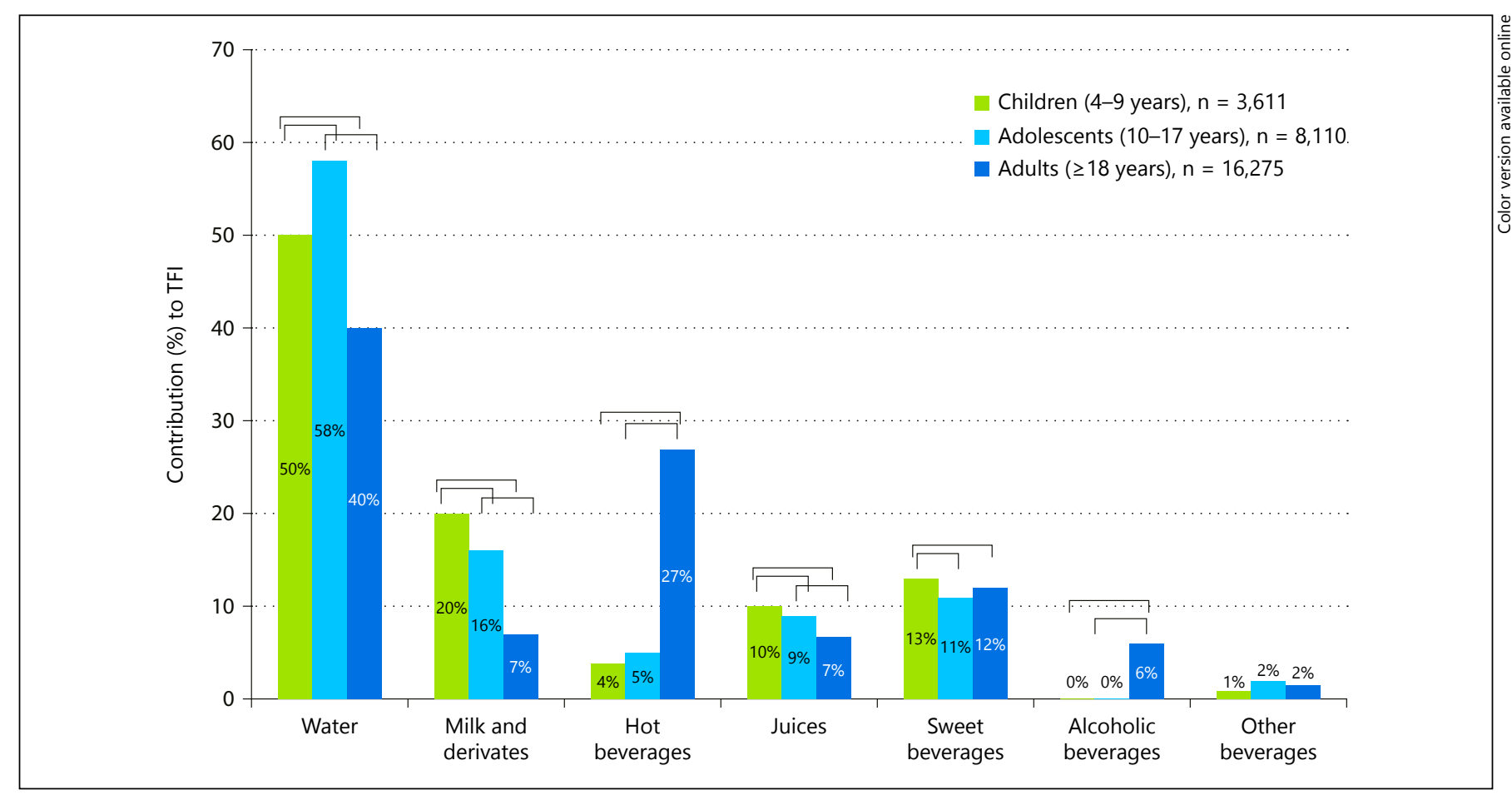

Fig. 3. Contribution (\%) of different fluid types to TFI according to age groups. Significant group differences (all values $\mathrm{p}<0.0001)$ are indicated by brackets. Diet beverages were not statistically compared between the age groups, due to missing data in the age groups $4-9$ and $10-17$ years.

samples of these 4 countries, the contribution of hot beverages was lower than in adults, yet juices and sweet beverages combined to contribute equally or more than water to the TFI. This also existed in the children and adolescent samples of Uruguay, Brazil and Mexico, where the highest contribution to TFI came from beverages containing sugar (i.e., juices and sweet beverages).

Figure 3 presents the overall contribution of water and different beverages to TFI, in the entire combined sample categorized by age groups. By far, water was the greatest single contributor to overall fluid intake, ranging from $40 \%$ of intake in adults to $57 \%$ in adolescents. With increasing age, the contribution of milk and derivatives, juices and sweet beverages significantly decreased, while the contribution of hot beverages and alcoholic beverages significantly increased (all values $\mathrm{p}<0.0001$ ).

\section{Discussion and Conclusion}

Our large, cross-sectional survey of 15 countries presented important aspects of habitual fluid intake by approximately 12,000 children and adolescents and
16,300 adults, using a similar fluid-specific record. The initial finding was that, on an average, a considerable proportion of participants in all age groups failed to meet the EFSA recommended adequate fluid intake [25]. Looking at the whole population, $47 \%$ of adults, $60 \%$ of children aged $4-9$ years and $75 \%$ of adolescents fell short of the recommended mean intake specific to their age group. While we recognize that we did not collect any marker of hydration status in this population, the fact that the reported intake was below the recommended intake raises a note of caution for public health policies, aimed at promoting the needs and benefits of sufficient daily fluid intake for all demographic groups.

A second finding, which has been repeatedly reported in other reviews [12], involves the wide variation found among fluid consumption by similar population age groups, across different countries. In our study, however, this difference may not be attributed to differences in the methodology to assess fluid intake, as has been the case in other published studies. We believe that these findings actually reveal different patterns of consumption, some of which may be explained by environmental differences, 
some of which respond to lifestyle and some of which may be due to differences in the diet and culture. These factors, in turn, also affect the type of beverages consumed by the population. Understanding the pattern of beverage consumption by different age groups within specific countries is a key element in fostering consumption of healthier beverages $[13,26]$.

The field of epidemiological descriptions of fluid intake at a population level is relatively new in the literature and presents several limitations. Our study was no exception, and we acknowledge the following limitations that should be addressed in future surveys using Liq. In $^{7}$ methodology. Fluid and water consumption, as well as food and nutrition surveys, are subject to sampling bias that reflects the underlying characteristics of the populations who respond to surveys. Furthermore, there are biases in self-reported data that require validation using biological markers [27]. Not all age groups are present in all surveys, in particular at-risk groups at the extremes of the age distribution; both young children and senior adults have been recognized to be at particular risk of inadequate fluid intake. Given that total water intake represents the sum of TFI and water present in foods and is subject to age, gender, body composition and diet/behavior/culture, it is suggested that the overall diet should be assessed to properly assess total water intake. Yet a potential risk, when recording food intake, is that subjects might be more focused on meals and omit drinking acts outside of meals. The evaluation of the contribution of free sugars present in beverages to overall energy consumption is still difficult, as reflected by the difficulties in assessing sugar added by hand to beverages, as well as the lack of updated fluid composition data in every country. In addition to difficulties in estimating overall energy intake from the diet and adequacy of energy intake, anthropometric data should be collected to look at energy balance.

Considering these limitations, we consider that our data contribute important information to the study of fluid consumption among populations. To the best of our knowledge, this is the largest study to collect information with a homogenized fluid-specific survey in a large sample of children, adolescents and adults. As such, the data offer a more robust picture of patterns of fluid consumption and types of beverages consumed across a wide spectrum of demographic groups. Understanding drinking behavior is the first step in the use of evidence to guide policies and programs, aimed at the promotion of healthier life styles.

\section{Disclosure Statement}

J.G., H.M., J.S.-S., L.A.M. and S.A.K. are members of the fluid intake expert group of Danone Research. S.A.K., J.S.-S. and L.A.M. have received research grants from Danone Research. I.G. is fulltime employee of Danone Research.

\section{References}

1 Daniels MC, Popkin BM: Impact of water intake on energy intake and weight status: a systematic review. Nutr Rev 2010;68:505521.

2 Schwartz AE, Leardo M, Aneja S, Elbel B: Effect of a school-based water intervention on child body mass index and obesity. JAMA Pediatr 2016;170:220-226.

3 Su SB, Wang JN, Lu CW, Guo HR: Reducing urinary tract infections among female clean room workers. J Womens Health (Larchmt) 2006; 15:870-876.

4 Roussel R, Fezeu L, Bouby N, Balkau B, Lantieri O, Alhenc-Gelas F, Marre M, Bankir L; D.E.S.I.R. Study Group: Low water intake and risk for new-onset hyperglycemia. Diabetes Care 2011;34:2551-2554.

5 Strippoli GF, Craig JC, Rochtchina E, Flood VM, Wang JJ, Mitchell P: Fluid and nutrient intake and risk of chronic kidney disease. Nephrology (Carlton) 2011;16:326-334.

6 Clark WF, Sontrop JM, Macnab JJ, Suri RS, Moist L, Salvadori M, Garg AX: Urine volume and change in estimated GFR in a communi- ty-based cohort study. Clin J Am Soc Nephrol 2011;6:2634-2641.

7 Sontrop JM, Dixon SN, Garg AX, BuendiaJimenez I, Dohein O, Huang SH, Clark WF: Association between water intake, chronic kidney disease, and cardiovascular disease: a cross-sectional analysis of NHANES data. Am J Nephrol 2013;37:434442.

8 Malik VS, Popkin BM, Bray GA, Després JP, Willett WC, Hu FB: Sugar-sweetened beverages and risk of metabolic syndrome and type 2 diabetes: a meta-analysis. Diabetes Care 2010;33:2477-2483.

9 Dhingra R, Sullivan L, Jacques PF, Wang TJ, Fox CS, Meigs JB, D’Agostino RB, Gaziano JM, Vasan RS: Soft drink consumption and risk of developing cardiometabolic risk factors and the metabolic syndrome in middleaged adults in the community. Circulation 2007; 116:480-488.

10 Jayalath VH, de Souza RJ, Ha V, Mirrahimi A, Blanco-Mejia S, Di Buono M, et al: Sugarsweetened beverage consumption and inci- dent hypertension: a systematic review and meta-analysis of prospective cohorts. Am J Clin Nutr 2015;102:914-921.

11 Ferraro PM, Taylor EN, Gambaro G, Curhan GC: Soda and other beverages and the risk of kidney stones. Clin J Am Soc Nephrol 2013;8: 1389-1395.

12 Özen AE, Bibiloni Mdel M, Pons A, Tur JA: Fluid intake from beverages across age groups: a systematic review. J Hum Nutr Diet 2015;28:417-442.

13 Elmadfa I, Meyer AL: Patterns of drinking and eating across the European Union: implications for hydration status. Nutr Rev 2015; 73(suppl 2):141-147.

14 Duffey KJ, Huybrechts I, Mouratidou T, Libuda L, Kersting M, De Vriendt T, et al: Beverage consumption among European adolescents in the HELENA study. Eur J Clin Nutr 2012;66:244-252.

15 Feferbaum R, de Abreu LC, Leone C: Fluid intake patterns: an epidemiological study among children and adolescents in Brazil. BMC Public Health 2012;12:1005. 
16 Fenández-Alvira JM, Iglesia I, Ferreira-Pêgo C, Babio N, Salas-Salvadó J, Moreno LA: Fluid intake in Spanish children and adolescents; a cross-sectional study. Nutr Hosp 2014;29: 1163-1170.

17 Ferreira-Pêgo C, Babio N, Fenández-Alvira JM, Iglesia I, Moreno LA, Salas-Salvadó J: Fluid intake from beverages in Spanish adults; cross-sectional study. Nutr Hosp 2014;29: 1171-1178.

18 Ferreira-Pêgo C, Guelinckx I, Moreno LA, Kavouras SA, Gandy J, Martinez H, et al: Total fluid intake and its determinants: crosssectional surveys among adults in 13 countries worldwide. Eur J Nutr 2015;54(suppl 2): $35-43$.
19 Guelinckx I, Ferreira-Pêgo C, Moreno LA, Kavouras SA, Gandy J, Martinez H, et al: Intake of water and different beverages in adults across 13 countries. Eur J Nutr 2015;54 (suppl 2):45-55.

20 Guelinckx I, Iglesia I, Bottin JH, De MiguelEtayo P, González-Gil EM, Salas-Salvadó J, et al: Intake of water and beverages of children and adolescents in 13 countries. Eur J Nutr 2015;54(suppl 2):69-79.

21 Iglesia I, Guelinckx I, De Miguel-Etayo PM, González-Gil EM, Salas-Salvadó J, Kavouras SA, et al: Total fluid intake of children and adolescents: cross-sectional surveys in 13 countries worldwide. Eur J Nutr 2015;54(suppl 2):57-67.

22 Ma G, Zhang Q, Liu A, Zuo J, Zhang W, Zou $S$, et al: Fluid intake of adults in four Chinese cities. Nutr Rev 2012;70(suppl 2):S105-S110.
23 Martinez H: Fluid intake in Mexican adults; a cross-sectional study. Nutr Hosp 2014;29: 1179-1187.

24 Senterre C, Dramaix M, Thiébaut I: Fluid intake survey among schoolchildren in Belgium. BMC Public Health 2014;14:651.

25 EFSA Panel on Dietetic Products, Nutrition and Allergies (NDA): Scientific opinion on dietary reference values for water. EFSA Journal 2010;8:1459.

26 Duffey KJ, Davy BM: The healthy beverage index is associated with reduced cardiometabolic risk in US adults: a preliminary analysis. J Acad Nutr Diet 2015;115:1682-1689.e2.

27 Armstrong LE: Assessing hydration status: the elusive gold standard. J Am Coll Nutr 2007;26(5 suppl):575S-584S. 